\title{
Regulation of growth hormone biosynthesis by Cdk5 regulatory subunit associated protein 1-like 1 (CDKAL1) in pituitary adenomas
}

\author{
Yoshihiro Takesue ${ }^{1), 2)}$, Fan-Yan Wei ${ }^{1)}$, Hiroyuki Fukuda ${ }^{1)}$, Yuki Tanoue ${ }^{3)}$, Takahiro Yamamoto ${ }^{1), 2)}$, \\ Takeshi Chujo ${ }^{1)}$, Naoki Shinojima ${ }^{2}$, Shigetoshi Yano $^{2), 4)}$, Motohiro Morioka ${ }^{5)}$, Akitake Mukasa ${ }^{2)}$, \\ Junichi Kuratsu ${ }^{2), 6)}$ and Kazuhito Tomizawa ${ }^{1), 7)}$ \\ 1) Department of Molecular Physiology, Faculty of Life Sciences, Kumamoto University, Kumamoto 860-8556, Japan \\ 2) Department of Neurosurgery, Faculty of Life Sciences, Kumamoto University, Kumamoto 860-8556, Japan \\ 3) International Research Center for Medical Sciences, Faculty of Life Sciences, Kumamoto University, Kumamoto 860-8556, Japan \\ 4) Department of Neurosurgery, Fukuoka Neurosurgical Hospital, Fukuoka 811-1313, Japan \\ 5) Department of Neurosurgery, Kurume University School of Medicine, Fukuoka 830-0011, Japan \\ 6) Department of Neurosurgery, Sakurajuji Hospital, Kumamoto 861-4173, Japan \\ 7) Center for Metabolic Regulation of Healthy Aging, Faculty of Life Sciences, Kumamoto University, Kumamoto 860-8556, Japan
}

\begin{abstract}
CDK5 regulatory subunit associated protein 1-like 1 (CDKAL1) is a tRNA-modifying enzyme that catalyzes 2methylthiolation $\left(\mathrm{ms}^{2}\right)$ and has been implicated in the development of type 2 diabetes (T2D). CDKAL1-mediated $\mathrm{ms}^{2}$ is important for efficient protein translation and regulates insulin biosynthesis in pancreatic cells. Interestingly, an association between T2D and release of growth hormone (GH) has been reported in humans. However, it is unknown whether CDKAL1 is important for hormone production in the pituitary gland. The present study investigated the role of CDKAL1 in GHproducing pituitary adenomas (GHPAs). CDKAL1 activity was suppressed in GHPAs, as evidenced by a decrease in $\mathrm{ms}^{2}$, compared with non-functioning pituitary adenomas (NFPAs), which do not produce specific hormones. Downregulation of Cdkall using small interfering and short hairpin RNAs increased the biosynthesis and secretion of GH in rat GH3 cells. Depletion of Cdkall increased the cytosolic calcium level via downregulation of DnaJ heat shock protein family (Hsp40) member $\mathrm{C} 10$ (Dnajc10), which is an endoplasmic reticulum protein related to calcium homeostasis. This stimulated transcription of GH via upregulation of Pit-1. Moreover, CDKAL1 activity was highly sensitive to proteostatic stress and was upregulated by suppression of this stress. Taken together, these results suggest that dysregulation of CDKAL1 is involved in the pathogenesis of GHPAs, and that modulation of the proteostatic stress response might control CDKAL1 activity and facilitate treatment of GHPAs.
\end{abstract}

Key words: tRNA, Hypophyseal tumor, Pituitary tumor, Growth hormone, Protein synthesis

\section{CDK5 REGULATORY SUBUNIT-ASSOCIATED} PROTEIN 1-LIKE $1(C D K A L 1)$ is a risk gene for the development of type 2 diabetes (T2D) [1-4]. Genetic variations in the intronic region of CDKAL1 are associated with decreased insulin secretion [5]. For instance, rs7756992 is one of the most reproducible T2Dassociated single-nucleotide polymorphisms (SNPs) [1]. Insulin secretion is $22 \%$ lower in homozygous carriers of the risk alleles (GG) of rs7756992 than in noncarriers

Submitted Dec. 3, 2018; Accepted May 6, 2019 as EJ18-0536 Released online in J-STAGE as advance publication Jun. 11, 2019 Correspondence to: Kazuhito Tomizawa, Department of Molecular Physiology, Faculty of Life Sciences, Kumamoto University, 1-1-1 Honjyo, Chuo-Ku, Kumamoto 860-8556, Japan.

E-mail: tomikt@kumamoto-u.ac.jp
[1]. The odds ratio for development of T2D in homozygous carriers of rs7756992 is 1.5 1.55 in European and Hong Kong populations.

The molecular function of CDKAL1 and its relevance to T2D have been elucidated using Cdkall-knockout mice $[6,7]$. CDKAL1 is a tRNA-modifying enzyme that specifically recognizes $\mathrm{tRNA}^{\mathrm{Lys}(\mathrm{UUU})}$ as a substrate and converts $N^{6}$-threonylcarbamoyladenosine $\left(\mathrm{t}^{6}\right)$ into 2methylthio- $N^{6}$-threonylcarbamoyladenosine $\left(\mathrm{ms}^{2} \mathrm{t}^{6} \mathrm{~A}\right)$ at position A37 adjacent to the anticodon [8]. The modified site can directly interact with the first nucleotide of Lys codons (AAA or AAG). This interaction stabilizes codon-anticodon binding and contributes to accurate and efficient decoding of Lys codons [6]. In Cdkallknockout mice, insulin biosynthesis is impaired at Lys 
codons, which causes aberrant insulin processing and ultimately leads to decreased insulin secretion and glucose intolerance [6]. Similar to the mouse model, CDKAL1 activity is decreased in individuals carrying the risk alleles of rs7756992 [9]. Furthermore, low CDKAL1 activity is associated with decreased insulin secretion in humans [9].

Various hormones, such as growth hormone $(\mathrm{GH})$, modulate the action of insulin [10]. GH is a multifunctional hormone that is secreted from the anterior lobe of the pituitary gland and acts on various tissues to stimulate metabolism and cell growth [10]. GH directly induces insulin release by pancreatic $\beta$-cells [11]. On the other hand, GH also elicits diabetogenic effects by inhibiting glucose uptake and increasing the blood glucose level $[12,13]$. Neoplasms arising from GH-producing pituitary adenomas (GHPAs) are characterized by excessive GH secretion, leading to the development of acromegaly $[14,15]$. Interestingly, abnormal secretion of GH in GHPAs is occasionally accompanied by T2D [16, 17]. For example, a previous study has shown that approximately $55 \%$ of patients with acromegaly have T2D or impaired glucose tolerance [18]. Moreover, $0.6 \%$ of hospitalized T2D patients were reported to have pituitary adenomas and mild acromegalic features [18].

Although CDKAL1 is ubiquitously expressed, the function of CDKAL1 in endocrine cells is unclear. Given the fundamental role of tRNA modification in the regulation of protein synthesis, we investigated whether CDKAL1 is involved in secretion of GH. Here, we show that downregulation of CDKAL1 increases secretion of $\mathrm{GH}$.

\section{Materials and Methods}

\section{Patients}

Tissues samples of GHPAs, non-functioning pituitary adenomas (NFPAs), and adrenocorticotropic hormoneproducing pituitary adenomas (ACTH-PAs) were collected during neurosurgeries at Kumamoto University Hospital in 1999-2014 and 2012-2014, 2007-2014, respectively. Informed consent was obtained from each patient before surgery. All procedures were approved by the Institutional Review Board (IRB) of Kumamoto University (Approval number: 231).

\section{Cell culture}

Rat GH3 cells (JCRB9047) were purchased from the JCRB Cell Bank. Cells were cultured in Ham's F10 medium (Thermo Fisher Scientific, Waltham, MA) containing 15\% horse serum (Thermo Fisher Scientific) and $2.5 \%$ fetal bovine serum (Corning, Corning, NY), and were maintained at $37^{\circ} \mathrm{C}$ in $5 \% \mathrm{CO}_{2}$ and $95 \%$ air.

\section{Knockdown of Cdkal1}

To knockdown Cdkall using small interfering RNA (siRNA), cells were transfected with Cdkall-targeting Silencer Select pre-designed siRNA (Cdkall siRNA; s167039; Ambion, Thermo Fisher Scientific) using RNAiMAX (Thermo Fisher Scientific) according to the manufacturer's instructions. To knockdown Cdkall using short hairpin RNA (shRNA), an oligonucleotide targeting Cdkall (5'-GCTTGCTGCCTATGGCTATAA-3') was cloned between the AgeI and EcoRI sites of the pLKO.1puro plasmid (Addgene, Watertown, MA) according to the manufacturer's instructions. pLKO.1-puro containing scrambled shRNA (shScramble; Sigma-Aldrich Japan, Tokyo, Japan) was used as a negative control. Lentiviral particles were generated by co-transfecting 293FT cells with the packaging vectors psPAX2 and pMD2.G using TransIT-LT1 Transfection Reagent (Mirus Bio, Madison, WI).

\section{Quantitative real-time PCR}

Total RNA was extracted using TRIzol reagent (Thermo Fisher Scientific) according to the manufacturer's instructions. cDNA was synthesized using PrimeScript RT Master Mix (Takara Bio, Kusatsu, Shiga, Japan). Quantitative real-time PCR was performed using TB Green Premix Ex Taq II (Takara Bio) according to the manufacturer's instructions. Primers used for the relative quantification are shown in Supplementary Table 1. The expression levels of human CDKAL1 and rat Cdkal1 were normalized to housekeeping genes $18 \mathrm{~S}$ rRNA. The expression levels of $X B P 1$ and ATF4 were normalized to the geometric mean of the levels of GAPDH, GUSB, or HPRT1. Data were analyzed by a ddCt method as described elsewhere.

\section{Analysis of 2-methylthiolation $\left(\mathrm{ms}^{2}\right)$}

The level of $\mathrm{ms}^{2}$ at position A37 of tRNA ${ }^{\text {Lys(UUU) }}$ was measured by a quantitative PCR-based method as described previously [19]. Briefly, total RNA was isolated from tumors or cultured cells using TRIzol. Two

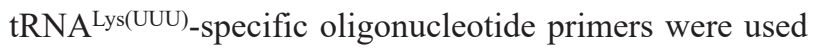
in the reverse transcription reaction: reverse primer $\mathrm{r} 1$, which was complementary to the region including A37, and reverse primer $r 2$, which was complementary to the downstream region of A37. Total RNA was mixed with 20 pmol of primer $\mathrm{r} 1$ or primer $\mathrm{r} 2$, and reverse transcription was initiated by adding 0.5 units of recombinant reverse transcriptase (Transcriptor First Strand cDNA Synthesis Kit; Roche Applied Science, Germany). An aliquot of the synthesized cDNA was mixed with a tRNA $^{\text {Lys(UUU) }}$-specific forward primer and reverse primer $\mathrm{r} 1$, and then subjected to quantitative PCR using the SYBR premix Ex Taq Kit (Takara). For an individual 
sample, the threshold cycle number was obtained by quantitative PCR using a cDNA template that was synthesized using reverse primer $\mathrm{r} 1$ or primer $\mathrm{r} 2$ (referred to as $\mathrm{C} \operatorname{Tr} 1$ or $\mathrm{CTr} 2$ ). The difference between $\mathrm{CTr} 1$ and CTr2 was calculated by subtracting CTr1 from CTr2 and was proportional to the level of $\mathrm{ms}^{2}$ [19]. The following primers were used to detect $\mathrm{ms}^{2}$ in tRNA ${ }^{\mathrm{Lys}(\mathrm{UUU})}$ : forward primer, GTCGGTAGAGCATCAGACTT; reverse primer r1, CCTGGACCCTCAGATTAAAA; reverse primer r2, GAACAGGGACTTGAACCCTG.

\section{Western blotting}

Cells were collected and lysed in RIPA buffer (1 M Tris-HCl, pH 8.0; $0.5 \% \mathrm{NP}-40 ; 150 \mathrm{mM} \mathrm{NaCl}$; and protease inhibitors) and sonicated for $10 \mathrm{~s}$. The protein concentration was determined using a BCA protein assay kit (Thermo Fisher Scientific). Samples were electrophoresed in $12 \%$ polyacrylamide gels and transferred to polyvinylidene fluoride membranes. Membranes were incubated with anti-GH (AF1067; Bio-techne, Minneapolis, MN), anti-CDKAL1 (sc-135456; Santa Cruz Biotechnology, Dallas, TX), anti-DnaJ heat shock protein family (Hsp40) member C10 (Dnajc10; 13101-1-AP; Proteintech, Rosemont, IL), anti-Sarco/Endoplasmic Reticulum Ca-ATPase 2 (SERCA2; ab3625; Abcam, Cambridge, MA), and anti-Pit-1 (sc-25258; Santa Cruz Biotechnology) antibodies diluted in phosphate-buffered saline (PBS) overnight at $4^{\circ} \mathrm{C}$. Signals were detected using Amersham ECL Prime Western Blotting Detection Reagent (GE Healthcare, Little Chalfont, Buckinghamshire, UK).

\section{Immunofluorescence microscopy}

Coverslips were coated with 10\% Matrigel matrix (Corning) prepared in PBS (Thermo Fisher Scientific) at $37^{\circ} \mathrm{C}$ for $1 \mathrm{~h}$. GH3 cells treated with siRNA or shRNA were seeded onto the coverslips, fixed with $4 \%$ paraformaldehyde (FUJIFILM Wako Pure Chemical, Osaka, Japan), stained with phalloidin (Thermo Fisher Scientific) to label actin, and observed under a confocal microscope (FV3000; Olympus, Tokyo, Japan).

\section{Enzyme-linked immunosorbent assay (ELISA) of rat $\boldsymbol{G H}$}

Cell culture medium was collected and diluted 1:100 with sterilized distilled water. The level of GH in the medium was analyzed using a Rat Growth Hormone ELISA Kit (BioVender, Karasek, Brno, Czech Republic) according to the manufacturer's instructions.

\section{Intracellular calcium imaging}

The calcium level in GH3 cells was monitored using a Calcium Kit-Fluo 4 (Dojindo, Mashiki, Kumamoto,
Japan). Cells were washed thrice with PBS, loaded with $2.5 \mu \mathrm{M}$ Fluo-4 for $30 \mathrm{~min}$, and then washed again with PBS. Fluo-4 fluorescence was observed using a FV3000 confocal microscope.

\section{Electron microscopy}

Tumor samples were collected during surgery, immediately fixed with $2 \%$ paraformaldehyde and $2 \%$ glutaraldehyde as described previously [6], and imaged by transmission electron microscopy.

\section{Statistical analysis}

All data were analyzed using GraphPad Prism 6 software. Data are expressed as the mean \pm standard error of the mean. Two groups were compared using the Student's $t$ test. Multiple groups were compared using a one-way analysis of variance, and then two groups were compared using the Student's $t$ test. A two-tailed $p$-value less than 0.05 was considered significant.

\section{Results}

\section{CDKAL1-mediated $\mathrm{ms}^{2}$ is decreased in GHPAs}

We investigated CDKAL1 activity by measuring the level of $\mathrm{ms}^{2}$ in total RNA isolated from growth hormoneproducing pituitary adenomas (GHPAs) and nonfunctioning pituitary adenomas (NFPAs) using a quantitative PCR-based method (Fig. 1A). The modification index represents the level of $\mathrm{ms}^{2}$ in cytosolic tRNA $^{\text {Lys(UUU) }}$. The level of $\mathrm{ms}^{2}$ was significantly lower in GHPAs than in NFPAs (Fig. 1A). By contrast, expression of $C D K A L 1$, which encodes the enzyme that mediates this modification, did not significantly differ between GHPAs and NFPAs (Fig. 1B). In addition, we analyzed the levels of $\mathrm{ms}^{2}$ and CDKAL1 mRNA in ACTH-PAs. Similar to GHPAs, the level of $\mathrm{ms}^{2}$ was significantly lower in ACTH-PAs than in NFPAs (Supplementary Fig. 1). The level of CDKAL1 mRNA did not differ between ACTH-PAs and NFPAs (Supplementary Fig. 1).

Next, we examined whether the rs7756992 SNP of CDKAL1 is associated with this difference in the level of $\mathrm{ms}^{2}$ between GHPAs and NFPAs. rs7756992 is one of the most reproducible T2D-related SNPs [1]. The risk alleles (AG and GG) of rs7756992 are associated with the development of T2D as well as a decrease in $\mathrm{ms}^{2}$ [9]. The number of patients carrying these risk alleles (AG and GG) did not significantly differ between those with GHPAs and those with NFPAs (Fig. 1C). Nevertheless, the level of $\mathrm{ms}^{2}$ was significantly lower in GHPAs than in NFPAs for each genotype (Fig. 1D). We did not analyze the genotype effect in ACTH-PAs because the sample size was small (rs7756992: $\mathrm{AA}=2, \mathrm{AG}=2, \mathrm{GG}=$ 4). Taken together, these results suggest that a decrease 

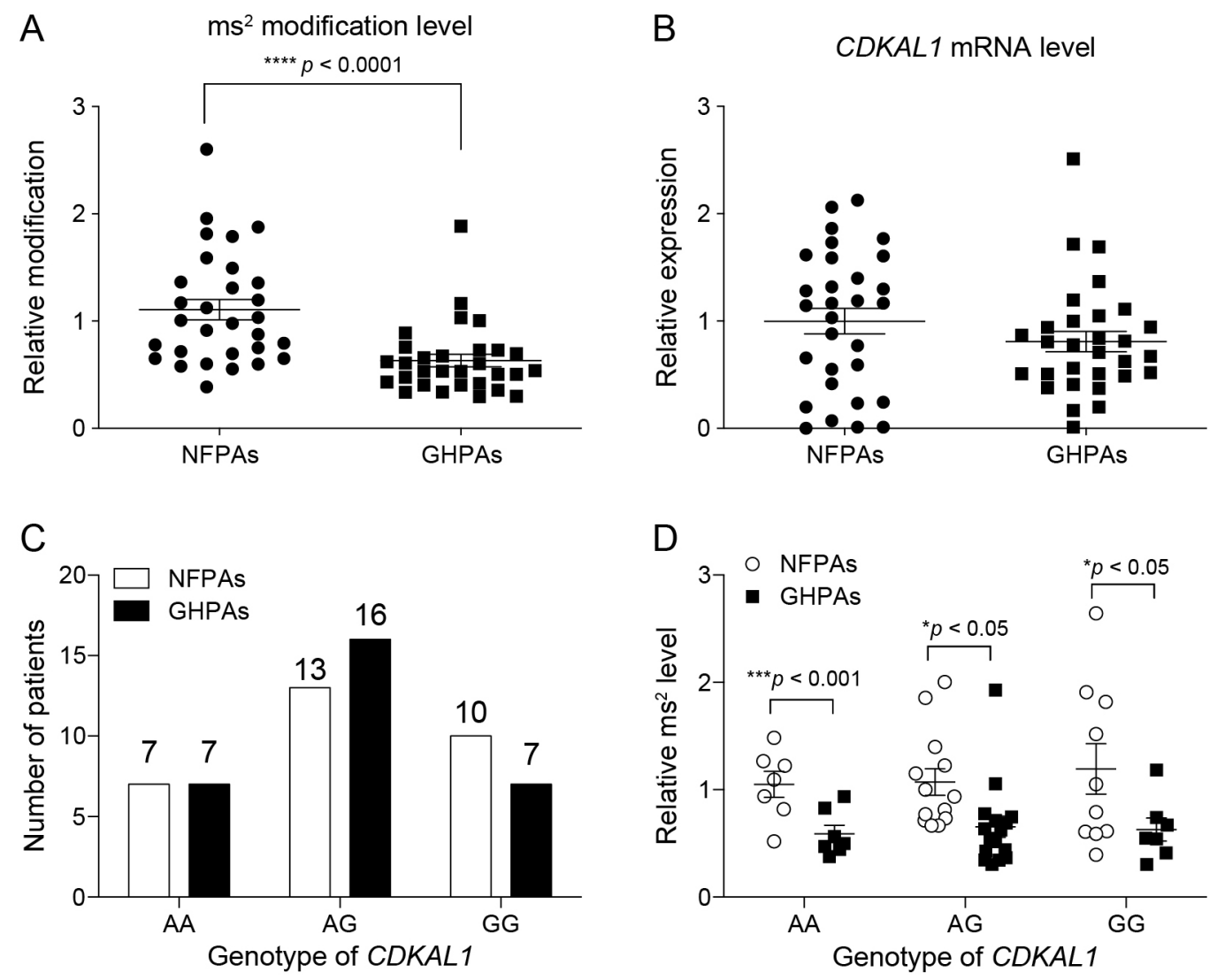

Fig. 1 The level of CDKAL1-mediated $\mathrm{ms}^{2}$ is decreased in GHPAs

(A) The level of CDKAL1-mediated $\mathrm{ms}^{2}$ in NFPAs and GHPAs extracted from patients was measured using a quantitative PCRbased method. Note that the level of $\mathrm{ms}^{2}$ is significantly decreased in GHPAs. $n=30$ per group. ${ }^{* * *} p<0.0001$. (B) The mRNA level of $C D K A L 1$ was compared between NFPAs and GHPAs. $n=30$ per group. (C) The numbers of patients carrying risk and non-risk alleles of CDKAL1 (rs7756992) are shown. Note that the G allele is the T2D-risk allele. (D) The level of $\mathrm{ms}^{2}$ in NFPAs and GHPAs of patients with various genotypes are shown. ${ }^{*} p<0.05,{ }^{* * *} p<0.001$.

in the $\mathrm{ms}^{2}$ level is involved in the pathogenesis of GHPAs; however, this decrease is unlikely due to the risk genotype of CDKAL1 or a reduction in mRNA expression of CDKAL1.

\section{Knockdown of Cdkal1 increases GH biosynthesis}

To elucidate the effect of CDKAL1 downregulation in GHPAs, we knocked down Cdkall in rat GH3 pituitary tumor cells by transfecting Cdkall-targeting siRNA (Cdkall siRNA). Transfection of Cdkall siRNA significantly decreased mRNA expression of Cdkall (Fig. 2A) and the level of $\mathrm{ms}^{2}$ (Fig. 2B) in these cells. Western blotting demonstrated that the protein level of $\mathrm{GH}$ was markedly higher in cells transfected with Cdkall siRNA than in cells transfected with control siRNA (Fig. 2C). In addition, cell culture medium was subjected to an ELISA to examine the effect of Cdkall on the secretion of GH. The level of GH was significantly higher in the culture medium of cells transfected with Cdkall siRNA than in the culture medium of cells transfected with control siRNA (Fig. 2D).
Next, we generated lentivirus carrying Cdkalltargeting shRNA (shCdkal1). mRNA expression of Cdkall and the level of $\mathrm{ms}^{2}$ were significantly lower in cells infected with the lentivirus carrying shCdkall than in cells infected with the control lentivirus carrying shScramble (Supplementary Fig. 2A and B). Accordingly, shRNA-mediated knockdown of Cdkall decreased and increased the protein levels of CDKAL1 and GH in GH3 cells, respectively (Fig. 3A). Consistently, the level of GH was significantly higher in the culture medium of cells infected with the lentivirus carrying shCdkall than in the culture medium of cells infected with the lentivirus carrying shScramble (Fig. 3B). Furthermore, immunocytochemistry demonstrated that the fluorescence intensity of GH-containing granules was higher in cells infected with the lentivirus carrying shCdkall than in cells infected with the lentivirus carrying shScramble (Fig. 3C). Thus, knockdown of Cdkall increases GH biosynthesis in $\mathrm{GH} 3$ cells. 

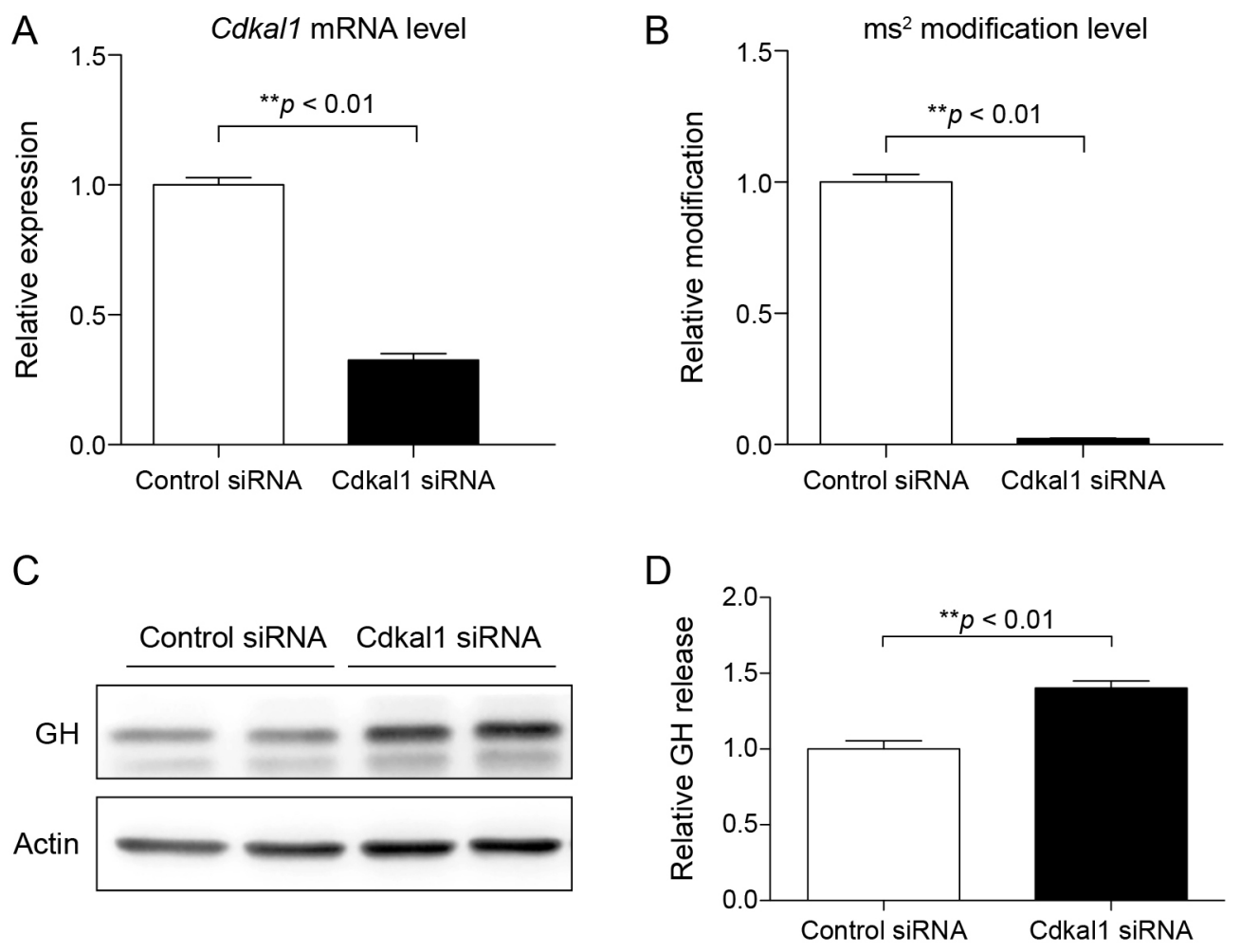

Fig. 2 siRNA-mediated knockdown of Cdkall increases GH biosynthesis in GH3 cells

(A) Relative mRNA expression of Cdkall in GH3 cells transfected with control or Cdkall siRNA was measured by quantitative PCR. $n=6$ per group. ${ }^{*} p<0.01$. (B) The level of $\mathrm{ms}^{2}$ was measured in GH3 cells transfected with control or Cdkall siRNA. Note that knockdown of Cdkall significantly decreased the level of $\mathrm{ms}^{2} . n=6$ per group. ${ }^{* *} p<0.01$. (C) The protein level of GH in GH3 cells transfected with control or Cdkall siRNA was measured by Western blotting. Note that knockdown of Cdkall increased the protein level of GH. (D) The level of GH in the culture medium of GH3 cells transfected with control or Cdkal1 siRNA was measured using an ELISA, and normalized against the cell number. $n=6$ per group. ${ }^{* *} p<0.01$.

\section{Knockdown of Cdkal1 alters calcium signaling}

Next, we investigated the molecular pathway by which downregulation of Cdkall increases GH biosynthesis. A complex mechanism involving calcium signaling regulates the biosynthesis of GH [20]. Thus, we used the Fluo-4 calcium indicator to determine the cytosolic calcium level in GH3 cells in which Cdkal1 had been downregulated using siRNA (Fig. 4A and B) or shRNA (Fig. $4 \mathrm{C}$ and $\mathrm{D}$ ). The basal cytosolic calcium level was significantly higher in cells transfected with Cdkall siRNA than in cells transfected with control siRNA. Under normal conditions, the majority of cytosolic calcium is transported into the endoplasmic reticulum (ER) by SERCA proteins, and consequently the cytosolic calcium level remains low [21]. SERCA2, a member of the SERCA family, is ubiquitously expressed in organs and tissues of mammals. Dnajc10 (also known as Erdj5) physically interacts with SERCA2, and regulates the calcium pump function of SERCA2 in a redox-dependent manner [22]. Western blotting demonstrated that the protein level of Dnajc10, but not SERCA2, was markedly decreased in cells infected with the lentivirus carrying
shCdkal1 (Fig. 4E and F). Calcium not only triggers release of $\mathrm{GH}$ but also enhances its transcription via Pit-1, which is the major transcription factor for pituitary hormones [23]. Consistently, knockdown of Cdkall increased the protein level of Pit-1 (Fig. 4F), resulting in upregulation of $G H$ mRNA (Fig. 4G). These results suggest that upregulation of $\mathrm{GH}$ in Cdkal1-knockdown GH3 cells is partly due to altered calcium signaling.

\section{Proteostatic stress regulates the function of CDKAL1}

We finally investigated the molecular mechanism underlying the differential regulation of CDKAL1 activity in GHPAs and NFPAs. In general, synthesis of large amounts of secretory proteins inevitably leads to production of aberrant proteins, which causes proteostatic stress in secretory cells [24]. In contrast with NFPAs, the majority of protein synthesis in GHPAs is dedicated to biosynthesis of GH [15]. Electron microscopy demonstrated that the ER was markedly dilated in the pituitary tumor cells from biopsies of GHPAs, which is indicative of proteostatic stress (Fig. 5A). By contrast, the ER had a normal morphology or was moderately dilated in pitui- 
A

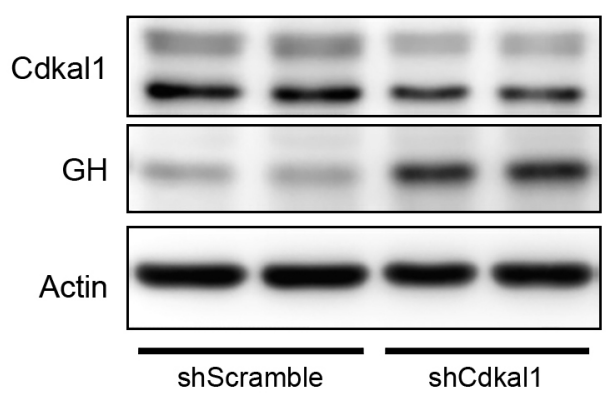

B

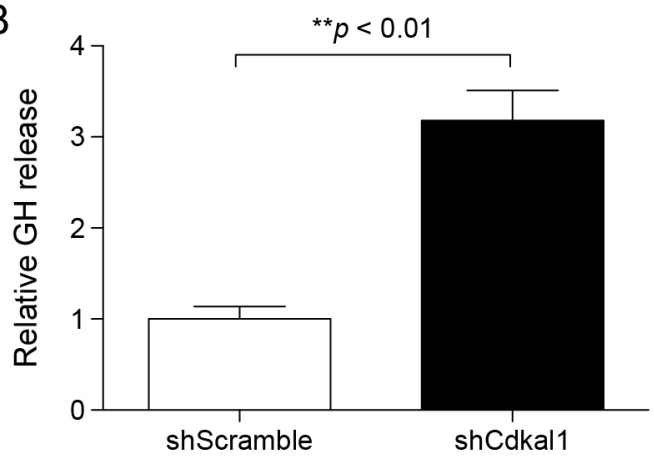

C
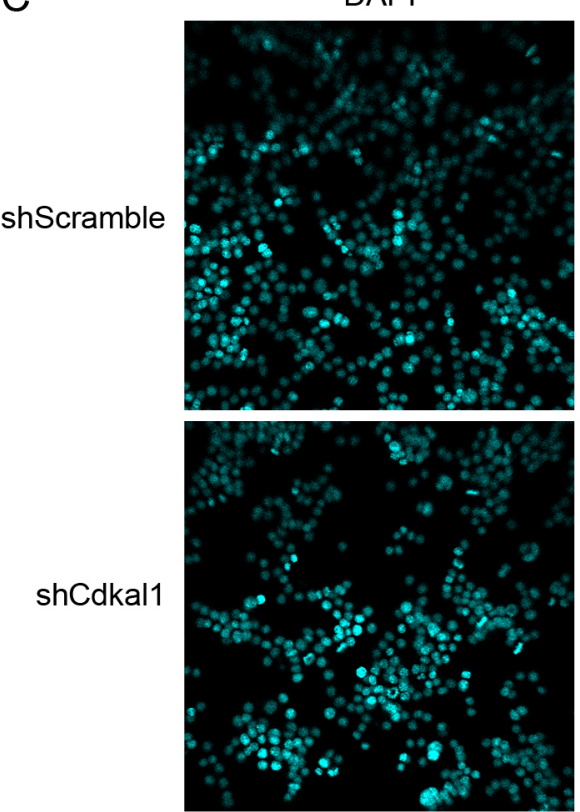

$\mathrm{GH}$
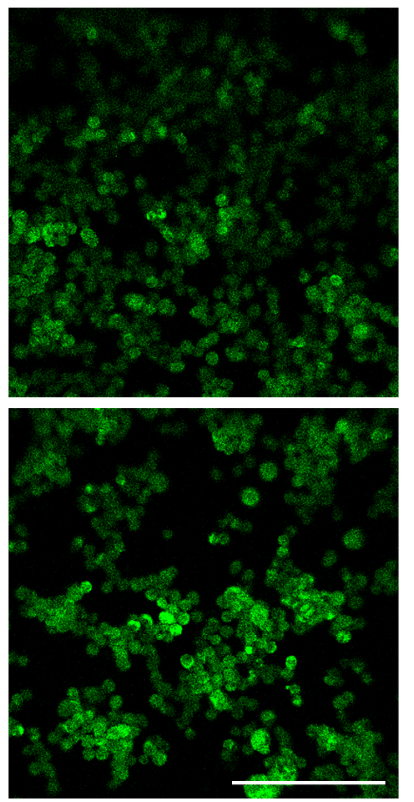

$\mathrm{GH}(\mathrm{x} 5)$
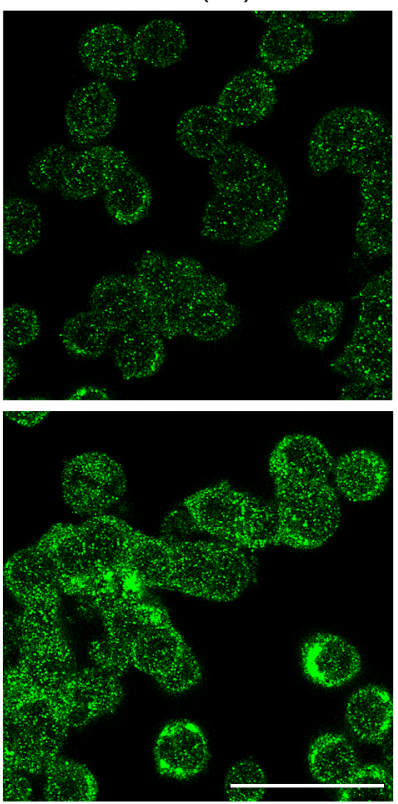

Fig. 3 shRNA-mediated knockdown of Cdkall increases GH biosynthesis in GH3 cells

(A) The protein levels of Cdkall and GH in GH3 cells infected with lentivirus carrying shCdkall or shScramble were examined by Western blotting. (B) The level of GH in the culture medium of GH3 cells infected with a lentivirus carrying shCdkall or shScramble was measured using an ELISA, and normalized against the cell number. $n=6$. $* * p<0.01$. (C) Immunofluorescence staining of GH was performed in GH3 cells infected with lentivirus carrying shCdkall or shScramble. Fluorescence corresponding to GH staining was strong and punctate in Cdkall-knockdown cells. Magnified images are shown on the right. Bars $=200 \mu \mathrm{m}$ (middle panel) and $40 \mu \mathrm{m}$ (right panel).

tary tumor cells from biopsies of NFPAs (Fig. 5A). Consistently, the expression levels of genes related to the proteostatic stress response, such as XBPI and ATF4, were significantly higher in GHPAs than in NFPAs (Fig. $5 B)$. Furthermore, the expression level of $X B P 1$ was negatively associated with the level of $\mathrm{ms}^{2}$ (Fig. 5C). These results suggest that proteostatic stress is closely correlated with the decrease in CDKAL1 activity. Finally, to examine whether proteostatic stress is capable to affect CDKAL1 activity, we treated GH3 cells with tunicamycin (TM) and thapsigargin (TG) to induce proteostatic stress. Treatment with TM and TG significantly decreased the level of $\mathrm{ms}^{2}$ (Fig. 5D). Conversely, treat- ment with Sal003, which prevents proteostatic stress by inhibiting dephosphorylation of eukaryotic initiation factor 2 subunit $\alpha$, significantly increased the level of $\mathrm{ms}^{2}$ (Fig. 5D). TM, TG, and Sal003 did not affect the expression level of Cdkall in GH3 cells (Supplementary Fig. 3 ); therefore, these reagents likely altered the $\mathrm{ms}^{2}$ level via a post-transcriptional mechanism.

\section{Discussion}

The present study reveals the molecular function of the tRNA-modifying enzyme CDKAL1 in the pathogenesis of GHPAs. CDKAL1 activity was suppressed in 
A
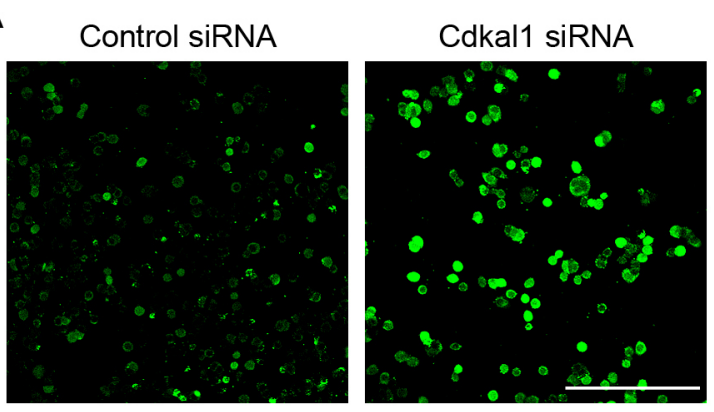

C

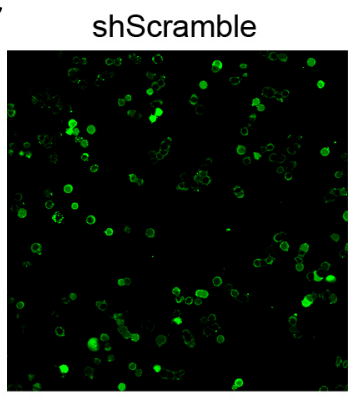

shCdkal 1

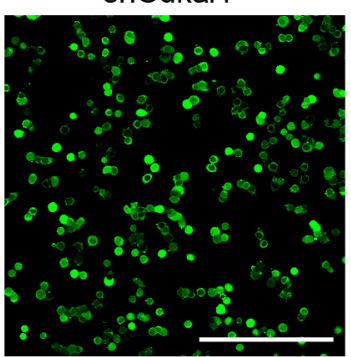

$\mathrm{B}$

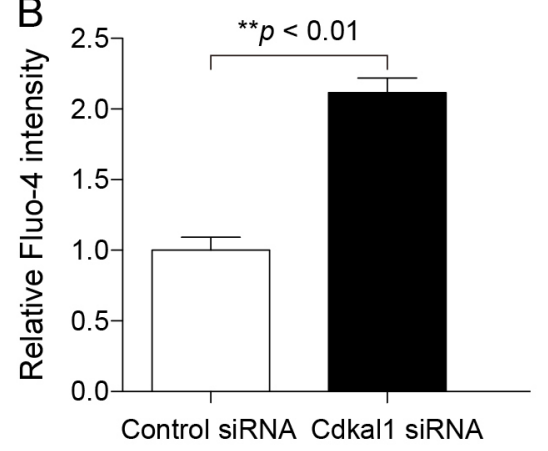

D

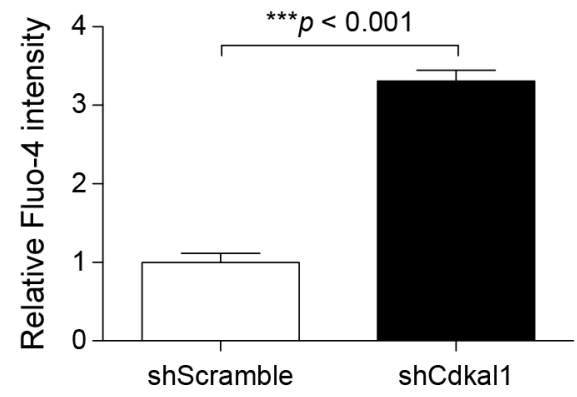

G

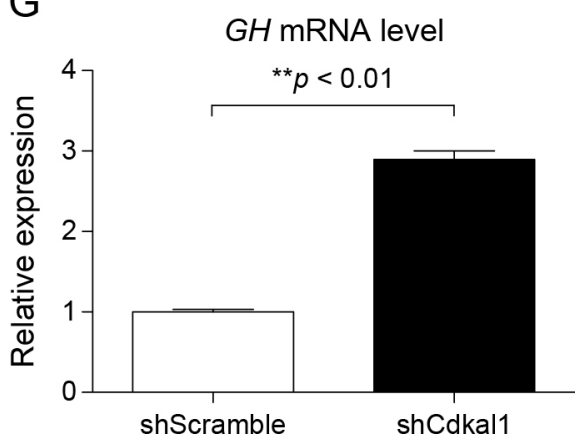

Fig. 4 Knockdown of Cdkall alters calcium homeostasis in GH3 cells

(A and B) GH3 cells were transfected with control or Cdkal1 siRNA. Intracellular calcium was visualized using Fluo-4 (A). Bar = $200 \mu \mathrm{m}$. The fluorescence intensity of Fluo-4 was quantified (B). $n=4,{ }^{* *} p<0.01$. (C and D) GH3 cells were infected with lentivirus carrying shScramble or shCdkall. Intracellular calcium was visualized using Fluo-4 (C). Bar $=200 \mu \mathrm{m}$. The fluorescence intensity of Fluo-4 was quantified (D). $n=6,{ }^{* * *} p<0.001$. (E-F) Protein levels of Dnajc10, SERCA2, Pit-1, and GH in GH3 cells infected with lentivirus carrying shScramble or shCdkall were examined by Western blotting. (G) mRNA expression of $G H$ was significantly increased in GH3 cells infected with lentivirus carrying shCdkall. $n=6, * * p<0.01$.

GHPAs due to proteostatic stress. Knockdown of Cdkall increased the biosynthesis and secretion of GH in GH3 cells. Downregulation of Cdkal1 suppressed expression of Dnajc10, which increased the cytosolic calcium level, leading to upregulation of $G H$ expression through Pit-1.

An important finding of this study is that downregulation of CDKAL1 in pituitary adenomas was associated with increased biosynthesis of GH. However, upregulation of GH secretion upon knockdown of Cdkall is surprising at the molecular level because loss of CDKAL1 perturbs $\mathrm{ms}^{2}$ of tRNA ${ }^{\mathrm{Lys}(\mathrm{UUU})}$, leading to impaired insulin biosynthesis in mouse and human pancreatic $\beta$-cells [6, 9]. Proinsulin contains two Lys residues, one of which is required for cleavage of this protein to generate mature insulin. Deficiency of CDKAL1 causes mistranslation of Lys, which impairs proinsulin processing and thereby decreases the mature insulin content. On the other hand, GH is cleaved between Ala26 and Phe27 to generate the mature form [25]. Thus, CDKAL1 dysfunction likely has less impact on production of GH due to the differential significance of Lys translation in hormone processing.

The present study showed that Cdkall is involved in 
A

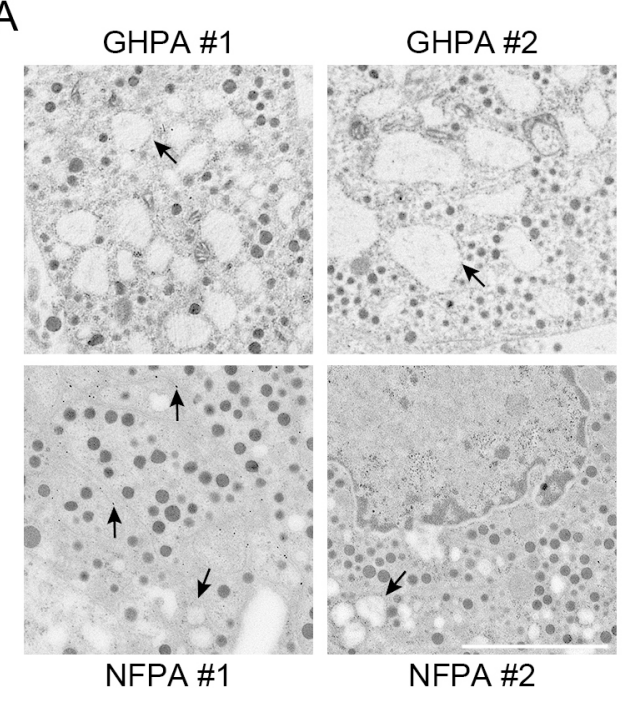

B
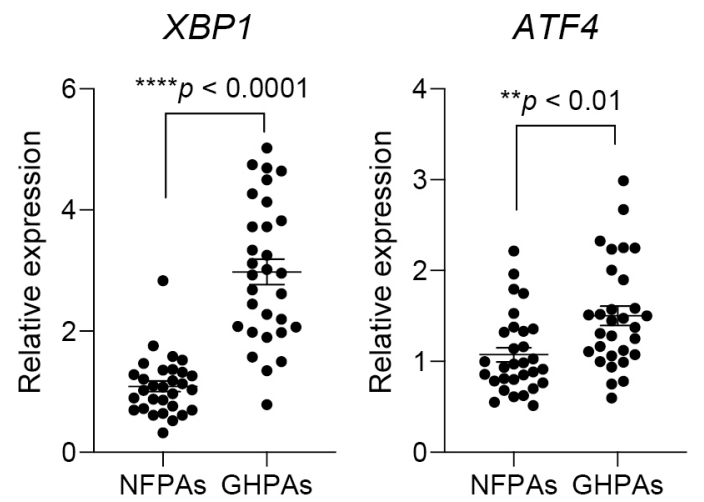

C

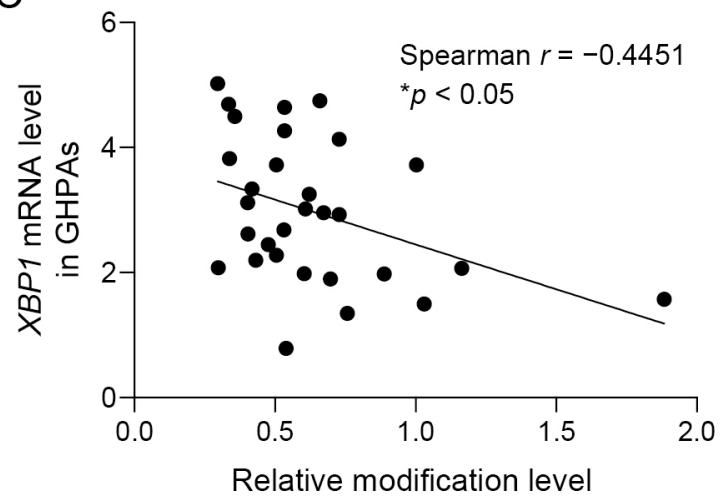

$\mathrm{D}$

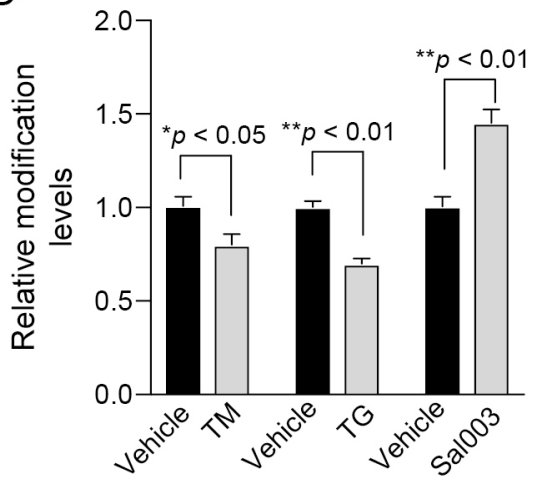

Fig. 5 Proteostatic stress regulates CDKAL1 activity

(A) Electron micrographs of GHPAs and NFPAs are shown. White arrows indicate dilated ER structures. Note that GHPA\#1 and GHPA\#2 were derived from two patients, while NFPA\#1 and NFPA\#2 were derived from one patient. Black arrows indicate normal ER structures. Bar $=2 \mu \mathrm{m}$. (B) Expression levels of XBP1 and ATF4 in NFPAs and GHPAs were examined by quantitative PCR. $n=30$ per group. ${ }^{* *} p<0.01, * * * * p<0.0001$. (C) The expression level of $X B P 1$ was negatively associated with the level of $\mathrm{ms}^{2}$ in GHPAs. (D) The level of $\mathrm{ms}^{2}$ was measured in GH3 cells treated with $10 \mu \mathrm{M} \mathrm{TM}, 10 \mu \mathrm{M}$ TG, or $2 \mu \mathrm{g} / \mathrm{mL}$ Sal003. $n=6$ per group, ${ }^{*} p<0.05,{ }^{* *} p<0.01$.

the regulation of intracellular calcium homeostasis through Dnajc10, which functions in GH biosynthesis. Dnajc10 is a disulfide reductase located in the ER and is the master regulator of calcium homeostasis in a redoxdependent manner [22, 26]. Specifically, Dnajc10 directly reduces the disulfide bond of SERCA2, leading to influx of calcium from the cytosol into the ER. Dysfunction of Dnajc10 or SERCA2 alters calcium homeostasis [22, 27]. Consistently, knockdown of Cdkal1 markedly reduced the protein level of Dnajc10, which was associated with an increased level of cytosolic calcium. Notably, Dnajc10 is highly expressed in secretory cells, including those in the hypothalamus, brain stem, and adrenal gland of mice [26, 28]. According to a human RNA database, the level of Dnajc10 transcripts is high in the pituitary gland and low in the pancreas. These results suggest that dysregulation of Dnajc10 upon downregulation of CDKAL1 is involved in the pathogenesis of GHPAs. A further study is required to investigate whether Dnajc10 is controlled at transcriptional or posttranslational level by CDKAL1.

$\mathrm{Ca}^{2+}$ is a major second messenger that not only controls exocytosis of hormones but also regulates gene expression. In the pituitary gland, $\mathrm{Ca}^{2+}$ is absolutely required for release of $\mathrm{GH}$ [20]. Moreover, an increase in $\mathrm{Ca}^{2+}$ is associated with upregulation of $G H$ expression in GH3 cells [29]. Various transcription factors, including Pit-1 and CREB, regulate transcription of GH. Importantly, $\mathrm{Ca}^{2+}$ is required for Pit-1-mediated transcription in GH3 cells [30]. Consistently, knockdown of Cdkal1 increased the level of Pit-1 in GH3 cells, which was associated with increased mRNA expression of $G H$. 
This study revealed that the proteostatic stress status regulates CDKAL1 activity in pituitary cells. In general, tRNA modifications are considered to be static [31]. However, CDKAL1-mediated $\mathrm{ms}^{2}$ is rapidly reactive to proteostatic stress. The level of $\mathrm{ms}^{2}$ is decreased by proteostatic stress and increased by inhibition of this stress. Proteostatic stress is usually triggered by protein misfolding in the ER, which induces various cellular responses at the translational and transcriptional levels [32]. Notably, proteostatic stress is closely coupled with the oxidative stress response because reactive oxygen species are usually generated as byproducts during protein folding [32]. Interesting, iron-sulfur clusters and reactive sulfur species, which are highly sensitive to oxidative stress, are required for CDKAL1 activity [33]. Indeed, oxidative stress effectively suppresses CDKAL1 function, which might explain the reduction in CDKAL1 activity in GHPAs. A further study is required to elucidate the potential role of oxidative stress in the regulation of CDKAL1 activity during the pathogenesis of GHPAs. CDKAL1 activity was increased in GH3 cells treated with Sal003, which inhibits proteostatic stress. Interestingly, Sal003 effectively induces cell death in various cancer cells such as leukemia and glioblastoma cells, suggesting that proteostatic stress is a therapeutic target in GHPAs [34, 35].

GHPAs can be microscopically categorized into two types: densely and sparsely granulated [36]. Densely granulated pituitary adenomas are strongly immunopositive for GH and contain large secretory granules. On the other hand, sparsely granulated pituitary adenomas are weakly immunopositive for GH and only contain a few small granules. Given the high demand for biosynthesis of $\mathrm{GH}$, densely granulated pituitary adenomas are likely exposed to a high level of proteostatic stress, which might affect CDKAL1 activity. A further study is required to investigate whether CDKAL1 activity and the mRNA expression level of CDKAL1 are differentially regulated in densely and sparsely granulated pituitary adenomas.

Excess GH affects insulin sensitivity and can alter the function of pancreatic $\beta$-cells $[16,17]$. Chronic GH excess induces insulin resistance by promoting gluconeogenesis in the liver and suppressing glucose uptake in muscles. Importantly, prolonged hyperglycemia can exaggerate oxidative stress in pancreatic $\beta$-cells, leading to impairment of insulin secretion [37]. Given the susceptibility of CDKAL1 activity to oxidative stress, it is conceivable that chronic GH excess indirectly decreases CDKAL1 activity in pancreatic $\beta$-cells, which ultimately impairs biosynthesis and secretion of insulin.

In conclusion, we demonstrated that downregulation of CDKAL1 is involved in the pathogenesis of GHPAs. Proteostatic stress suppressed CDKAL1 activity, which led to dysregulation of calcium homeostasis via Dnajc10 downregulation and, consequently, upregulation of GH production.

\section{Acknowledgments}

We thank Nobuko Maeda (Kumamoto University) for providing technical assistance.

\section{Disclosure}

None of the authors report any conflicts of interest. This work was supported by a Grant-in-Aid for Scientific Research from the Ministry of Education, Culture, Sports, Sciences, and Technology of Japan [17905074 and 18959602 to $\mathrm{KT}, 18 \mathrm{H} 02599$ and $18 \mathrm{~K} 19521$ to FYW], the Japan Agency for Medical Research and Development (AMED) [17935694 to KT], and the Takeda Science Foundation [KT].

\section{References}

1. Steinthorsdottir V, Thorleifsson G, Reynisdottir I, Benediktsson R, Jonsdottir T, et al. (2007) A variant in CDKAL1 influences insulin response and risk of type 2 diabetes. Nat Genet 39: 770-775.

2. Zeggini E, Weedon MN, Lindgren CM, Frayling TM, Elliott KS, et al. (2007) Replication of genome-wide association signals in UK samples reveals risk loci for type 2 diabetes. Science 316: 1336-1341.

3. Diabetes Genetics Initiative of Broad Institute of Harvard and MIT, Lund University, and Novartis Institutes of BioMedical Research, Saxena R, Voight BF, et al. (2007) Genome-wide association analysis identifies loci for type 2 diabetes and triglyceride levels. Science 316: 1331-
1336.

4. Scott LJ, Mohlke KL, Bonnycastle LL, Willer CJ, Li Y, et al. (2007) A genome-wide association study of type 2 diabetes in Finns detects multiple susceptibility variants. Science 316: 1341-1345.

5. Wei FY, Tomizawa K (2018) tRNA modifications and islet function. Diabetes Obes Metab 20 Suppl 2: 20-27.

6. Wei FY, Suzuki T, Watanabe S, Kimura S, Kaitsuka T, et al. (2011) Deficit of tRNALys modification by Cdkall causes the development of type 2 diabetes in mice. $J$ Clin Invest 121: 3598-3608.

7. Ohara-Imaizumi M, Yoshida M, Aoyagi K, Saito T, Okamura T, et al. (2010) Deletion of CDKAL1 affects 
mitochondrial ATP generation and first-phase insulin exocytosis. PLoS One 5: e15553.

8. Arragain S, Handelman SK, Forouhar F, Wei FY, Tomizawa K, et al. (2010) Identification of eukaryotic and prokaryotic methylthiotransferase for biosynthesis of 2methylthio-N6-threonylcarbamoyladenosine in tRNA. $J$ Biol Chem 285: 28425-28433.

9. Zhou B, Wei FY, Kanai N, Fujimura A, Kaitsuka T, et al. (2014) Identification of a splicing variant that regulates type 2 diabetes risk factor CDKAL1 level by a codingindependent mechanism in human. Hum Mol Genet 23: 4639-4650.

10. Vijayakumar A, Yakar S, LeRoith D (2011) The intricate role of growth hormone in metabolism. Front Endocrinol (Lausanne) 2: 32.

11. Wu Y, Liu C, Sun H, Vijayakumar A, Giglou PR, et al. (2011) Growth hormone receptor regulates $\beta$ cell hyperplasia and glucose-stimulated insulin secretion in obese mice. J Clin Invest 121: 2422-2426.

12. Kilgour E, Baldwin SA, Flint DJ (1995) Divergent regulation of rat adipocyte GLUT1 and GLUT4 glucose transporters by GH. J Endocrinol 145: 27-33.

13. del Rincon JP, Iida K, Gaylinn BD, McCurdy CE, Leitner $\mathrm{JW}$, et al. (2007) Growth hormone regulation of $\mathrm{p} 85$ expression and phosphoinositide 3-kinase activity in adipose tissue: mechanism for growth hormone-mediated insulin resistance. Diabetes 56: 1638-1646.

14. Beckers A, Petrossians P, Hanson J, Daly AF (2018) The causes and consequences of pituitary gigantism. Nat Rev Endocrinol 14: 705-720.

15. Budan RM, Georgescu CE (2016) Multiple pituitary adenomas: a systematic review. Front Endocrinol (Lausanne) 7: 1 .

16. Giordano R, Guaraldi F, Berardelli R, Karamouzis I, D'Angelo V, et al. (2012) Glucose metabolism in patients with subclinical Cushing's syndrome. Endocrine 41: 415423.

17. Hannon AM, Thompson CJ, Sherlock M (2017) Diabetes in patients with acromegaly. Curr Diab Rep 17: 8.

18. Suda K, Fukuoka H, Iguchi G, Hirota Y, Nishizawa H, et al. (2015) The prevalence of acromegaly in hospitalized patients with type 2 diabetes. Endocr J 62: 53-59.

19. Xie P, Wei FY, Hirata S, Kaitsuka T, Suzuki T, et al. (2013) Quantitative PCR measurement of tRNA 2methylthio modification for assessing type 2 diabetes risk. Clin Chem 59: 1604-1612.

20. Chang JP, Habibi HR, Yu Y, Moussavi M, Grey CL, et al. (2012) Calcium and other signalling pathways in neuroendocrine regulation of somatotroph functions. Cell Calcium 51: 240-252.

21. Chemaly ER, Troncone L, Lebeche D (2018) SERCA control of cell death and survival. Cell Calcium 69: 46-61.

22. Ushioda R, Miyamoto A, Inoue M, Watanabe S, Okumura $\mathrm{M}$, et al. (2016) Redox-assisted regulation of $\mathrm{Ca}^{2+}$ homeo- stasis in the endoplasmic reticulum by disulfide reductase ERdj5. Proc Natl Acad Sci 113: E6055-E6063.

23. Hoggard N, Davis JRE, Berwaer M, Monget P, Peers B, et al. (1991) Pit-1 binding sequences permit calcium regulation of human prolactin gene expression. Mol Endocrinol 5: $1748-1754$.

24. Wong MY, DiChiara AS, Suen PH, Chen K, Doan ND, et al. (2018) Adapting secretory proteostasis and function through the unfolded protein response. Curr Top Microbiol Immunol 414: 1-25.

25. Li CH, Parkoff H (1956) Preparation and properties of growth hormone from human and monkey pituitary glands. Science 124: 1293-1294.

26. Cunnea PM, Miranda-Vizuete A, Bertoli G, Simmen T, Damdimopoulos AE, et al. (2003) ERdj5, an Endoplasmic Reticulum (ER)-resident protein containing DnaJ and thioredoxin domains, is expressed in secretory cells or following ER stress. J Biol Chem 278: 1059-1066.

27. Talukder MAH, Kalyanasundaram A, Zuo L, Velayutham M, Nishijima Y, et al. (2008) Is reduced SERCA2a expression detrimental or beneficial to postischemic cardiac function and injury? Evidence from heterozygous SERCA2a knockout mice. Am J Physiol Heart Circ Physiol 294: H1426-H1434.

28. Uhlen M, Fagerberg L, Hallstrom BM, Lindskog C, Oksvold P, et al. (2015) Tissue-based map of the human proteome. Science 347: 1260419.

29. Thorner MO, Holl RW, Leong DA (1988) The somatotrope: an endocrine cell with functional calcium transients. J Exp Biol 139: 169-179.

30. Tada M, Kobayashi H, Moriuchi T (1999) Molecular basis of pituitary oncogenesis. $J$ Neurooncol 45: 83-96.

31. Agris PF (2004) Decoding the genome: a modified view. Nucleic Acids Res 32: 223-238.

32. Rendleman J, Cheng Z, Maity S, Kastelic NI, Munschauer M, et al. (2018) New insights into the cellular temporal response to proteostatic stress. eLife 7: e39054.

33. Takahashi N, Wei FY, Watanabe S, Hirayama M, Ohuchi $\mathrm{Y}$, et al. (2017) Reactive sulfur species regulate tRNA methylthiolation and contribute to insulin secretion. Nucleic Acids Res 45: 435-445.

34. Drexler HCA (2009) Synergistic apoptosis induction in leukemic cells by the phosphatase inhibitor salubrinal and proteasome inhibitors. PLoS One 4: e4161.

35. Dadey DYA, Kapoor V, Khudanyan A, Thotala D, Hallahan DE (2018) PERK regulates glioblastoma sensitivity to ER stress although promoting radiation resistance. Mol Cancer Res 16: 1447-1453.

36. Syro LV, Rotondo F, Ramirez A, Di Ieva A, Sav MA et al. (2015) Progress in the diagnosis and classification of pituitary adenomas. Front Endocrinol (Lausanne) 6: 97.

37. Bensellam M, Jonas JC, Laybutt DR (2018) Mechanisms of $\beta$-cell dedifferentiation indiabetes: recent findings and future research directions. $J$ Endocrinol 236: R109-R143. 